\title{
PAN-COLONIC PRESSURIZATIONS ASSOCIATED WITH RELAXATION OF THE ANAL SPHINCTER IN HEALTH AND DISEASE: A NEW COLONIC MOTOR PATTERN IDENTIFIED USING HIGH-RESOLUTION MANOMETRY
}

Maura Corsetti *^, Giuseppe Pagliaro*, Ingrid Demedts*, Eveline Deloose*, Annemie Gevers*, Charlotte Scheerens*, Nathalie Rommel*, and Jan Tack*.

Translational Research Center for Gastrointestinal disorders (TARGID)* Department of Clinical and Experimental Medicine University of Leuven

Leuven, Belgium

^ National Institute for Health Research, Nottingham Digestive Diseases Biomedical Research Unit, Nottingham University Hospitals NHS Trust, University of Nottingham, Nottingham, UK

Short title: Pan-colonic pressurizations in humans

Abbreviations: $\mathrm{HRM}=$ high-resolution manometry; $\mathrm{HAPS}=$ high-amplitude propagating sequences; $\mathrm{EMG}=$ electromyography; $\mathrm{VAS}=$ visual analogue scale; $\mathrm{SD}=$ standard deviation.

Word count: 5009

Postal Address: Jan Tack, M.D., Ph.D.

Department of Internal Medicine

Division of Gastroenterology

University Hospital Gasthuisberg

Herestraat 49

B-3000 Leuven, Belgium

Tel.: +3216 344225

Fax: +3216344419

E-mail:Jan.Tack@med.kuleuven.be

The data of the present manuscript have been presented as oral presentations at the First Federation of Neurogastroenterology and Motility Meeting in Guangzhou, China September 2014 (Neurogastroenterol Mot 2014; Issue 26: 9), at Digestive Disease Week (DDW) Washington, USA, May 2015 (Gastroenterology 2015; 148: S-192) and at Neurogastro 2015 Istanbul, Turkey, June 2015 (Neurogastroenterol Mot 2015; 27: S-34). 


\section{AUTHORS'CONTRIBUTIONS TO THE PAPER:}

MC designed the study, performed all the colonic manometry studies, made the quantitative and qualitative analysis of the data and wrote the manuscript.

GP helped to perform the quantitative and qualitative analysis of the data.

ED helped to perform the statistical analysis of the data.

NR contributed to the interpretation of data and provided critical revision of the manuscript

ID and AG performed colonoscopies to place the manometry catheter

CS contributed to the interpretation of data

JT designed the study, performed colonoscopies to place the catheter, contributed to the interpretation of data and provided critical revision of the manuscript.

Competing Interests: the authors have no competing interests.

Funding: This study was supported by a Methusalem Grant from K.U.Leuven to Prof Jan Tack and by the Fund for Scientific Research Flanders (FWO-Vlaanderen), as Maura Corsetti is a FWO Pegasus Marie Curie Fellow. 


\begin{abstract}
Background: Only few studies have applied high-resolution manometry (HRM) to the study of colonic motility in adults and none of them have concurrently evaluated colonic and anal motor activity. Aims: To evaluate colonic and anal motor activity by means of HRM in healthy subjects. As the present study revealed the presence of a new colonic motor pattern (pan-colonic pressurizations) in healthy subjects, three additional studies were conducted: the first and the second to exclude that this motor event results from an artefact due to abdominal wall contraction and to confirm its modulation by cholinergic stimulation and the third, as pilot study, to test the hypothesis that this colonic pattern is defective in patients with chronic constipation refractory to current pharmacological treatments. Methods: In both volunteers and patients the HRM catheter was advanced proximally during colonoscopy. Results: In all subjects, pressure increases of $15 \pm 3$ $\mathrm{mmHg}$ and $24 \pm 4 \mathrm{~s}$ simultaneously occurring in all colonic sensors (pan-colonic pressurizations), associated with anal sphincter relaxation were identified. Subjects had $85 \pm 38$ pan-colonic pressurizations which increased significantly during meal $(p=0.007)$ and decreased afterward $(p=0.01)$, and were correlated with feelings of and desire to evacuate gas. The mean number of propagating sequences was $47 \pm 39$, and only retrograde increased significantly postprandially $(p=0.01)$. Pan-colonic pressurizations differed from strain artifacts and significantly increased after prostigmine. In patients pan-colonic pressurizations were significantly reduced as compared to volunteers. Conclusions: Pan-colonic pressurizations associated with relaxations of the anal sphincter represent a new colonic motor pattern which seems to be defective in patients with treatment-refractory chronic constipation and may play a role in the transport of colonic gas and in the facilitation of the propagating sequences-induced colonic transport.
\end{abstract}

Keywords: pan-colonic pressurizations, colonic motility, anal relaxation, high-resolution manometry. 


\section{Study highlights}

\section{What is current knowledge}

- high-resolution manometry increases the accuracy in detecting colonic low-amplitude motor activity

- no studies with high-resolution manometry have evaluated the coordination between colonic and anal motor activity in adults

\section{What is new here}

- Concurrent evaluation of colonic and anal motor activity allowed to recognize pan-colonic pressurizations associated with anal sphincter relaxations as a new colonic motor event

- In healthy subjects the pan-colonic pressurizations are the main colonic motor pattern, increase significantly during the meal and decrease afterwards in concert with increased occurrence of mainly retrograde propagating colonic contractions.

- In patients with slow-transit chronic constipation refractory to any pharmacological treatment the pan-colonic pressurizations are significantly reduced as compared to healthy subjects. 


\section{INTRODUCTION}

Normal activity of the colon, reflected by bowel function, is critical for health and quality of life. Chronic idiopathic constipation is a functional disorder of the gastrointestinal tract, defined by the Rome III criteria as a condition with multiple symptoms, including straining, lumpy or hard stools, sensation of incomplete evacuation, sensation of anorectal obstruction and less than 3 bowel movements per week (1). The condition is common, with prevalence between $4 \%$ and $20 \%$ in cross-sectional community-based surveys (2). It has been demonstrated that the impact of chronic constipation on quality of life for patients is comparable to that of organic conditions, such as chronic obstructive pulmonary disease, diabetes and depression (3). Alterations of colonic motility have been implicated in the pathophysiology of chronic idiopathic constipation (1). Previous studies, applying manometry catheters with $7.5 \mathrm{~cm}$ spacing, have demonstrated that colonic motor activity in health is represented mainly by non-propagated activity, with propagated sequences of low amplitude and high amplitude occurring respectively only in numbers of $2.4 \pm 0.1$ and $0.4 \pm 0.1$ per hour $(4,5)$. Both non-propagated and propagated activity has been found to be reduced in patients with chronic idiopathic constipation (6). However, as there are no quantitative or qualitative data that unequivocally differentiate normal from abnormal colonic function $(7,8)$, the role of colonic manometry in the management of these patients is unclear (8) and, at the moment, it is recommended to confirm colonic inertia (absence of motor response to meal and of high-amplitude propagating sequences (HAPSs) after pharmacological stimulation, ie bisacodyl) in patients with slow-transit not responding to pharmacological treatments selected for surgery (8).

The introduction of high-resolution manometry (HRM) to the study of esophageal motility has improved the understanding of esophageal dysmotility and has led to a new classification of patients in different subtypes with different treatment outcomes $(9,10)$. In this technique, the number of sensors recording the intraluminal pressure has been increased to one every $1 \mathrm{~cm}$ compared to one every $5 \mathrm{~cm}$ with conventional manometry. HRM has only recently been introduced in colonic studies $(11,12)$ and it was found that the application of HRM to the study of colonic activity increases the accuracy in detecting low-amplitude propagating contractions (12).

At present only few studies have applied HRM to the study of colonic motility in adult humans, and these have been performed mainly in a single center using a fiber-optic catheter (12-14). As normal colonic function requires the coordination between colonic and anal motor activity and as none of the previous studies with HRM simultaneously assessed colonic and anal motor activity in adults, the aim of the present study was to use HRM to evaluate colonic motility and its relationship with anal sphincter activity during fasting and fed state in healthy subjects. Our hypothesis was that HRM could refine our understanding about normal colonic motility. As the present study revealed the presence of a new colonic motor pattern in healthy subjects, three additional studies have been performed: the first and the second to exclude that this colonic event results from an artefact due to 
abdominal wall contraction during strain and to confirm that it is modulated by cholinergic stimulation and the third, as pilot study, to test the hypothesis that this colonic pattern is defective in patients with chronic constipation refractory to laxatives, prucalopride, lubiprostone or linaclotide referred to our motility clinic for evaluation of possible colectomy. The choice of the cholinergic stimulus was based on the fact that prostigmine has been demonstrated to induce colonic contractions in previous studies $(15,16)$

\section{MATERIALS AND METHODS}

\section{Healthy Subjects and Patients}

In the main and additional studies 1 and 2, we studied respectively ten ( $30 \pm 11$ years, 5 females), three ( $28 \pm 2$ years, 2 females) and four (42 \pm 5 years, 2 females) healthy subjects with a normal bowel habit defined by presence of bowel movements between two per day to one every two days. None of the subjects had organic or functional disease affecting the gastrointestinal system. None had previous abdominal surgery other than appendectomy and none was taking laxatives or other medications. All the subjects of the main study also participated to a study with oral administration of bisacodyl and demonstrated at least one HAPS (data not shown) (17). In additional study 3 we studied ten patients ( $41 \pm 14$ years, all females) with refractory chronic constipation as defined according to Rome III criteria with a mean duration of $10 \pm 5$ years of symptoms. All the patients present a slow colonic transit as confirmed using radiopaque markers (18), none had organic diseases that affect the gastrointestinal system or a history of previous abdominal surgery other than appendectomy, and they all did not report any chronic intermittent abdominal pain or discomfort. All had received treatment with polyethylene glycol (either alone, $13.8 \mathrm{mg}$ twice or more a day, or in combination with bisacodyl or sodium picosulfate), prucalopride (2 mg/day), lubiprostone (24 $\mu \mathrm{g}$ b.i.d.) or linaclotide $(290 \mu \mathrm{g} / \mathrm{die})$ respectively for at least one month, in case of good tolerance, without satisfactory response. All the patients were using intermittent water enemas to defecate. Each healthy subject and patient gave his/her written informed consent prior to the study, which was approved by the Ethics Committee of the University Hospitals Leuven.

\section{Experimental procedure of the main study}

On the day of the experiment each healthy subject presented to the Motility Unit of the Gastroenterology Department, University Hospital of Leuven, in the early morning to undergo, after a bowel preparation with water enemas, a colonoscopy-assisted positioning of the colonic HRM catheter as previously described (19). A 10-F solid state HRM catheter comprising 40 pressure sensors spaced $2.5 \mathrm{~cm}$ apart (Unisensor AG, Switzerland) was used (Supplementary Figure 1). Under conscious sedation (midazolam $5 \mathrm{mg}$ i.v. and pethidine $50 \mathrm{mg}$ i.v.) the colonoscope with the attached HRM catheter was advanced as far as possible into the colon. After reaching the desired location the HRM catheter was clipped to the colonic mucosa, the colonoscope was gradually 
removed while maximally aspirating air from the colon, and the external part of the catheter was taped to the buttockof the subject. The position of the tip of the HRM catheter and the absence of gas distending the colon was confirmed fluoroscopically after colonoscopy and at the end of measurement. The subjects were then asked to lie on a bed and the external end of the catheter was connected to a recording system (Medical Measurement Systems, Enschede, Netherlands). The colonic pressure recording was started and continued for four hours before and three hours after a standardized meal $(313,8 \mathrm{kcal}$, two slices of white bread with cheese and butter and a vanilla pudding (11.6 g protein (14.7\%), $13.8 \mathrm{~g}$ fat (39.7\%) and $35.8 \mathrm{~g}$ carbohydrates $(45.6 \%))$. The choice of the caloric content of the meal was based on a usual Belgian lunch. During the first two hours of recording they were allowed to sleep (to recover from sedation) while in the remaining period they were asked to be awake. During the periods that the subjects were awake, they were asked to score every $15 \mathrm{~min}$ their feeling of abdominal gas, desire to evacuate gas, desire to defecate, urgency to defecate, abdominal discomfort or pain, or any other sensations on a $100 \mathrm{~mm}$ visual analogue scale (VAS).

Experimental procedure of Additional study 1: concomitant evaluation of colonic motility and abdominal wall electromyography (EMG) activity in healthy subjects

This study was conducted as the main one but, during the colonic motility measurement, the EMG activity was concurrently recorded at two different sites corresponding to the lower rectum on both sides of the abdomen. At each site, EMG activity was recorded by means of bipolar $\mathrm{Ag}-\mathrm{AgCl}$ surface electrodes (Kendall ${ }^{\mathrm{TM}}$ Covidien, Canada, USA) placed $3 \mathrm{~cm}$ apart. The electrodes were connected to an EMG recording system (Solar Wireless, Medical Measurement Systems, Enschede, Netherlands). The correct location of the electrodes was checked by recording EMG responses to maximal voluntary abdominal contraction.

Experimental procedure of Additional study 2: effect of cholinergic modulation on colonic motility in healthy subjects

In this study the colonic pressure recording was conducted for two hours during the recovery period, one hour during basal period, and one hour after the administration of the acetylcholinesterase inhibitor prostigmine $0.5 \mathrm{mg}$ iv (Prostigmine $\AA$, Meda Pharma, Brussels, Belgium). Blood pressure and heart rate were monitored every $15 \mathrm{~min}$ after prostigmine administration. During the period after administration of prostigmine each subject was instructed to report the onset of any sensations they might have felt during the recording period by means of an event marker and to score their feeling of the same sensations evaluated during the main study on the VAS scale. 
Experimental procedure of Additional study 3: pilot study to evaluate the presence and characteristics of pan-colonic pressurizations in a group of patients with chronic constipation refractory to current pharmacological treatments

The experimental procedure was the same as in the main study in healthy subjects, but in the patients an additional recording period was added at the end of the study, to evaluate during a 2hours period the response to intra-colonic administration of bisacodyl (Dulcolax®, Boehringer Ingelheim, Brussels, Belgium; 2 capsules, $10 \mathrm{mg}$, dissolved in $10 \mathrm{ml}$ of saline) through an infusion tube (Entral ${ }^{\mathrm{TM}}$, RT10/100, Eurosteriel Medical, Netherlands) attached at the tip of the HRM catheter. During the period after intra-colonic administration of bisacodyl, the patients were only asked to use the event marker to report their sensations.

\section{Data analysis}

As a first step artifacts (such as catheter movements, cough, body movements and strain) were identified and removed. Then the colon was divided in three anatomical regions: right colon (from cecum to splenic flexure), left colon (from splenic flexure to sigmoid colon) and rectum. Based on the fluoroscopic images, each recording sensor was then allocated to its closest anatomical region. The sensors located at the level of the anal sphincter were assigned to the anal sphincter region.

Potential pressure waves (monophasic pressure change with an onset to peak amplitude $>5$ $\mathrm{mmHg}$ ) were identified and those occurring in three or more adjacent channels defined as propagating sequences, according to previous definition $(5,13)$. Propagating sequences were qualified as anterograde (anally propagating) or retrograde (orally propagating) $(5,13)$ and further sub-classified as high-amplitude propagating sequences (HAPSs, if the amplitude of two of the propagating pressure waves was $\geq 100 \mathrm{mmHg}$ and that of another one was $\geq 80 \mathrm{mmHg}$ ) (20) or as low-amplitude propagating sequences in the remaining cases. Then low-amplitude sequences were defined as single, cyclic (repetitive propagating sequences), long distance or simultaneous (three or more pressure waves occurring simultaneously in adjacent recording sites) as previously reported $(13,21)$. Non-propagating activity (pressure changes not associated morphologically or temporally with pressure patterns recorded in adjacent sensors) were also recognised according to previous definitions (5).

Number of propagating and simultaneous sequences, amplitude ( $\mathrm{mmHg}$ ), duration (seconds), propagation extent (based on the number of sensors involved in the sequence, $\mathrm{cm}$ ) as well as the colonic site of origin (right colon, left colon or rectum) were evaluated. For each colonic motor pattern, the relation with the anal sphincter relaxation was evaluated. Applying the definitions previously reported during prolonged recording of the anal sphincter activity $(22,23)$, the anal sphincter relaxation was defined as a drop in the resting anal sphincter pressure (of at least 25 $\mathrm{mmHg}$ ) with subsequent quick return to basal values over the following $30-60$ seconds. The onset 
and the end, the percent of relaxation (from baseline to nadir) and the duration (seconds) of the anal relaxation were evaluated. A colonic motor pattern was defined as associated with sphincter relaxation if the onset of the anal sphincter relaxation started in the time period of the duration of the colonic motor event. In the main study resting sphincter pressure was also calculated as the mean pressure recorded by the sensors located at the level of the anal sphincter every 15 minutes. In addition an analysis of the colon motor activity was performed using computer software calculating the colonic motility index $(\mathrm{Ml}=[\ln$ (number of peaks $x$ the sum of peak amplitudes +1$)$ (24). This analysis was used as overall measure of the colonic motor activity and an approximate index of non-propagating activity. In the main and additional study 3, the VAS score of different sensations was also averaged per period of 15 minutes starting from the moment the subjects were awake after the recovery period.

\section{Statistical Analysis}

The outcome parameters were compared by means of Wilcoxon signed rank test when paired and by means of Mann-Whitney test when unpaired. Differences were considered to be significant at the $5 \%$ level and Bonferroni correction was applied to multiple comparisons. To analyze the relationship between different sensations and colonic motor patterns, multiple regression analysis was performed with sensations as the dependent variables and motor patterns as the independent variable. Mixed model analysis was used to analyze the effect of different recording periods and of different regions of the colon on the motility index, with time as a continuous independent variable and region as an independent categorical variable. All data are presented as mean \pm SD.

\section{RESULTS}

\section{Main study}

The manometry catheter tip was clipped to the proximal right colon in eight out of the 10 subjects and in the transverse colon in the remaining subjects. In none of the cases the catheter was displaced at the fluoroscopic control at the end of the study.

\section{Colonic motility index}

Table 1 in the Supplementary file reports the motility index of each region of the colon during the different recording periods. Using mixed models analysis, the motility index, which was used as overall measure of the colonic motor activity, increased from recovery to pre-prandial and to postprandial conditions in each region of the colon $(p=0.003)$ with a greater effect in the left colon ( $p=0.04$ after Bonferroni's correction).

\section{Colonic motor patterns}


During the recording, besides the propagating sequences and non-propagating activity, a never reported colonic motor pattern was noted in each subject and here named pan-colonic pressurization.

\section{Low-amplitude propagating sequences}

In all but one subject, low-amplitude propagating sequences with a mean total number of $47 \pm 39$ were recognized during the entire recording period. The number of both anterograde and retrograde sequences tended to increase from recovery to preprandial conditions (respectively, $1 \pm 2$ vs $8 \pm 4$ for anterograde, $p=0.16$ and $0.6 \pm 1$ vs $2 \pm 2$ for retrograde, $p=0.17$ ), and only the number of retrograde propagating sequences was significantly increased in the postprandial period $(14 \pm 7$ for retrograde, $p=0.01$ and $7 \pm 5$ for anterograde).

See Supplementary File for Subtypes of low-amplitude propagating sequences, Long distance lowamplitude propagating sequences and Non-propagating activity.

\section{HAPS}

Only one subject displayed two HAPS which occurred one hour after the consumption of the meal (Supplementary Figure 2C). The two HAPS appeared 90 seconds apart from each other and their origin was in the middle and distal transverse colon with an extent of propagation respectively of 22.5 and $30 \mathrm{~cm}$. The mean amplitude of the first was $172 \mathrm{mmHg}$ and that of the second 196 $\mathrm{mmHg}$. Both HAPS were associated with the occurrence of strong feeling of desire to defecate. The relationship with anal sphincter activity was evaluated only for the first HAPS as during the second the subject requested the commode to defecate. The amplitude of anal relaxation was $48 \%$ and its duration $30 \mathrm{~s}$.

\section{Pan-colonic pressurizations}

In all subjects the presence of pressure increases simultaneously occurring in all colonic sensors (pan-colonic pressurizations) and associated with anal sphincter relaxation were identified. Figure 1 shows a part of a tracing with three pan-colonic pressurizations associated with anal sphincter relaxation in one subject. The number of the pan-colonic pressurizations was $85 \pm 38$ per subject during the entire recording time. The first pan-colonic pressurization appeared within $40 \pm 39$ minutes after the start of recording and then they appeared with variable frequencies ranging from 1 every 3-4 minutes in most of the cases to 1 per minute in some cases (Figure 2). The number of pan-colonic pressurizations did not differ between recovery and preprandial conditions $(6 \pm 4 / 30 \mathrm{~min}$ vs $8.6 \pm 7 / 30 \mathrm{~min}, \mathrm{p}=0.57$ ) but it significantly increased during the meal $(19 \pm 9 / 30 \mathrm{~min}, \mathrm{p}=0.007)$ and decreased during the postprandial period $(2 \pm 2 / 30 \mathrm{~min}, \mathrm{p}=0.01)$ as compared to preprandial period. During meal, the frequency of these pan-colonic pressurizations was of $0.83 \pm 0.36$ events per minute. The amplitude and duration of each pressure increases were respectively $15 \pm 3 \mathrm{mmHg}$ and 
$24 \pm 4 \mathrm{~s}$ and at detailed analysis, the anal sphincter relaxations started $5 \pm 1 \mathrm{~s}$ after the starting of the pan-colonic pressurization and lasted $23 \pm 4 \mathrm{~s}$ with a relaxation of $56 \pm 23 \%$ (Figure 1).

See Supplementary File for Subtypes of Pan-colonic pressurizations, Relationship between pancolonic pressurizations and low-amplitude propagating sequences or non-propagating activity and for anal sphincter activity.

\section{Relationship between sensations and colonic motor patterns}

Figure $3 \mathrm{~A}$ shows the average VAS score for the sensation of feeling of gas, desire to evacuate gas, desire to defecate, urgency to defecate and discomfort/pain reported by each subject during the recording period from the moment they awaked after the recovery. As regards to the relationship between sensations and different colonic motor patterns, at multivariate analysis, there was a significant positive relationship between pan-colonic pressurizations and both feeling of gas $(\beta=0.45 \pm 0.21 ; p=0.03)$ and desire to evacuate gas $(\beta=0.60 \pm 0.24 ; p=0.01)$ (Figure 3B). No relationship was found between any of the other sensations and pan-colonic pressurizations, or between any sensations and propagating sequences.

\section{Additional study 1: concomitant evaluation of the colonic motility and of the abdominal wall} electromyography (EMG) activity in healthy subjects

The manometry catheter tip was clipped to the proximal right colon in two out of the 3 subjects and in the transverse colon in the remaining subject. In none of the cases was the catheter displaced at the fluoroscopic control at the end of the study. In all subjects pan-colonic pressurizations associated with anal sphincter relaxations were recorded with a mean number of $94 \pm 52$ per subjects. None of these pan-colonic events was associated with a concomitant increase in the abdominal EMG activity (Figure $4 A$ ). In the same subjects a mean number of $51 \pm 29$ artifacts due to cough, movements and strain were recorded. In all cases these artifacts were associated with simultaneous pressure increases in all the HRM sensors including those located at the level of the anal sphincter and with concomitant increase in the abdominal EMG activity (Figure 4A). Figure 4B show part of tracing in one subject with artifacts due to movement and one due to strain and with a pan-colonic pressurization. We recorded $50 \pm 1$ low-amplitude propagating sequences and no HAPSs in these subjects.

\section{Additional study 2: effect of cholinergic modulation on pan-colonic pressurizations in healthy subjects}

The manometry catheter tip was clipped to the proximal right colon in all four subjects. The administration of prostigmine did not affect blood pressure (106 \pm 1.5 vs $103 \pm 2.5 \mathrm{mmHg}$ for systolic pressure, $p=0.33$ and $59 \pm 3$ vs $59 \pm 6 \mathrm{mmHg}$ for diastolic pressure, $\mathrm{p}=0.97)$ or heart rate $(61 \pm 1.5$ vs $55 \pm 2$ bpm, $p=0.08$ ). None of the subjects developed cholinergic side effects. In all the subjects 
pan-colonic pressurizations associated with anal sphincter relaxations were recorded. The number of pan-colonic pressurizations did not differ between the recovery period and the pre-prandial period, while a significant increase occurred after the administration of prostigmine $(p=0.01)$ (Figure $5 \mathrm{~A})$. After prostigmine, the majority $(72 \pm 12 \%)$ of the pan-colonic pressurizations was associated with a number of sensations which attributed a mean VAS score of $59 \pm 17$ to feeling of gas, $57 \pm 24$ for desire to evacuate gas, $6 \pm 3$ for abdominal pain/discomfort, $0.54 \pm 0.22$ for desire to defecate, and zero for urgency to defecate. One subject had an episode of flatus associated with a pan-colonic pressurization at the end of the recording period after prostigmine. Figure 5B reports an example of the response to prostigmine in one subject. No HAPSs and $20 \pm 2$ low-amplitude propagating contractions were recorded in the subjects.

\section{Additional study 3: pilot study to evaluate the presence of pan-colonic pressurizations in a group of patients with chronic constipation refractory to pharmacological treatments}

The manometry catheter tip was clipped to the proximal right colon in eight patients and in the transverse colon in the remaining patient. The mean number of both pan-colonic pressurizations and low-amplitude propagating sequences was significantly reduced in patients with chronic refractory constipation as compared to healthy subjects (19 $\pm 21, P=0.009$ and $6 \pm 10, P=0.01$ respectively), none of these increased after the meal and no HAPSs were recorded in this patient group. In four patients no pan-colonic pressurizations were recorded and in one patient $45 \%$ of pan-colonic pressurizations were represented by interrupted ones. The majority of low-amplitude propagating sequences were anterograde (75 $\pm 10 \%)$. Supplementary Figure 6 reports the average VAS score for sensations reported by each patients during the recording period from the moment they awaked after the recovery. After a mean of $12 \pm 11 \mathrm{~min}$ from the intra-colonic administration of bisacodyl, atypical HAPSs as in figure 6A appeared in three patients, repetitive pan-colonic pressurizations as in figure $6 \mathrm{~B}$ and $6 \mathrm{C}$ appeared in two patients, and a combination of repetitive pan-colonic pressurizations and HAPSs in another patient (figure 7D). In two cases the occurrence of repetitive pan-colonic pressurizations coincided with partial expulsion of the catheter (figure 7B and 7D). Four patients did not show any change in motor pattern in response to the drug. The occurrence of repetitive pan-colonic pressurizations was associated with pain while HAPSs were connected with discomfort.

\section{DISCUSSION}

The present study describes for the first time the occurrence of a new colonic motor pattern in humans characterized by pan-colonic pressurizations associated with anal sphincter relaxation. In the present experimental conditions the pan-colonic pressurizations represent the main colonic motor pattern, increase significantly during the meal and decrease afterwards, in concert with increased occurrence of mainly retrograde propagating colonic contractions. They differ from 
artifacts due to abdominal wall contraction during strain and are significantly increased by the administration of acetylcholinesterase inhibitor prostigmine. In patients with chronic constipation not responding to laxatives, prucalopride, lubiprostone or linaclotide, the pancolonic pressurizations are significantly reduced as compared to healthy subjects.

Previous studies applying conventional manometry have reported the occurrence of simultaneous contractions in the colon in healthy subjects $(21,25-28)$. Their presence was to some extent also described in a study combining conventional manometry with colonic barostat measurements, as pressure increases simultaneously involving the intra-colonic barostat balloon in the proximal colon and the manometry recording points below (29). However, in the past, some authors have attributed these simultaneous pressure increases to the occurrence of artifacts of abdominal wall contraction during strain. In the present study, the concomitant evaluation of colonic and anal sphincter activity has allowed the distinction of these pan-colonic pressurizations, which are associated with anal sphincter relaxation, from artifacts or straining events, which are associated with simultaneous pressure increases also in the sensors located at the anal sphincter level. The concomitant evaluation of colonic motility and of abdominal EMG activity has further confirmed this distinction. Moreover the fact that the number of pan-colonic pressurizations is modulated by the same factors also influencing the other colonic motor patterns (meal) and by prostigmine, which has been previously reported to increase colonic tone and induce contractions recorded by the electronic barostat $(15,16)$, suggest they are generated by the activity of colonic wall.

Previous studies evaluating the motor activity of the rectum and of the anal sphincter during prolonged recording periods have described the occurrence of spontaneous relaxations of the anal sphincter with a frequency in fasting, prandial and postprandial period similar to the frequency of the anal sphincter relaxations here found to be associated with pan-colonic pressurizations $(22,23,30,31)$. In some of these studies the association with the presence of pressure increases in rectum was noticed $(30,31)$, but their association with synchronous pan-colonic motor activity has not been described before.

In the present study we studied the colonic motility and the effect of intra-colonic administration of bisacodyl for the first time in a group of chronic constipation patients, not responding to any of the current pharmacological therapies. This selected population of patients shows a significantly reduced number of pan-colonic pressurizations, which became the main motor pattern after bisacodyl in some patients and was associated with partial expulsion of the HRM catheter in two cases. These data are in line with previous studies evaluating, by means of the conventional manometry, the motor activity of the rectum and of the anal sphincter or of the colon during prolonged recording periods, which have reported a lower frequency of spontaneous anal relaxations (22) and of simultaneous colonic pressure rises in patients with chronic constipation compared to healthy subjects (32). Moreover, they are in line two previous studies, respectively 
applying the conventional and the HRM in adult and in children constipated patients, which noted the occurrence of simultaneous pressure increases after administration of bisacodyl $(33,34)$. In the first of the two studies, these pressure increases were also associated with transport of radioisotope (33). All these findings seem to suggest that pan-colonic pressurizations, which represent a frequent pattern in health, are defective in this subgroup of patients, but in some cases can be elicited by drug stimulation and even participate in the process of expulsion. However these data need to be confirmed in larger group of patients, also to assess whether in these patients the pharmacological modulation of pan-colonic pressurizations may represent a new therapeutic target.

The mechanism underlying the generation of pan-colonic pressurizations associated with anal sphincter relaxations needs to be determined. However, the comparison of their characteristics with those of the contractile events reported by several basic research papers shows that they share many features of the rhythmic contractility described to be generated by the human colon in vitro (35-38). This activity has been suggested to represent a normal response of the colon to even minimal distension and to play a role in facilitating the contact between the colonic wall and the intraluminal content to favor absorption, mixing but also propulsion (38). With regard to their possible role in propulsion, the fact that in the present study these pan-colonic events overlap with propagating sequences both in anterograde and retrograde direction, suggest that they may play a role in maintaining the colonic wall in a "tonic" contraction and facilitate the movement of colonic content induced by propagating sequences. This hypothesis has been suggested by a previous paper combining colon transit scintigraphy and conventional manometry (27). Moreover as in previous $(5,30,33,39)$ and in the present study, both spontaneous and prostigmine-induced pancolonic pressurizations were correlated with sensation of gas and desire to evacuate gas and some of these were associated with the actual occurrence of flatus, these observations may also suggest a potential venting function of pan-colonic pressurizations associated with anal sphincter relaxation similar to what demonstrated for transient lower esophageal sphincter relaxations. In this regard the "tonic" contraction induced by pan-colonic pressurizations and the associated anal sphincter relaxation could be sufficient to induce the movement of gas inside the colon. Given that the colon is main part of the gut where fermentation occurs and it contains about $60 \%$ of gas of the gut (40), it is indeed conceivable that a colonic venting system exists. Ongoing studies combining HRM catheter with impedance segments are evaluating whether these pan-colonic events are indeed associated with gas transport. Finally, even if the experimental conditions (ambulatory setting and request to score sensations every 15 minutes) could have enhanced the awareness of these pan-colonic events, which are likely to remain unperceived under physiological conditions, the fact that they can be perceived (as shown more clearly in the present study with prostigmine, and the fact their reduction coincides with almost complete absence of abdominal sensations in the present subgroup of patients) could suggest that they are involved in the induction of abdominal 
symptoms. Ongoing studies are evaluating their role in the subgroup of patients with constipation and abdominal pain.

With respect to propagating colonic contractions, the data of the present study in healthy subjects confirm those of previous studies performed with colonic fiber-optic HRM $(12,13)$. There are some numerical differences in occurrence of motor events in the present paper, which may be related to the meal used (low-caloric in the present study) and to the resolution of the catheter $(2.5 \mathrm{~cm}$ interval between measuring points as compared to $1 \mathrm{~cm}$ with the fiber-optic catheter). On the other hand, the studies applying the fiber optic catheter, to date, did not report the presence of pancolonic pressurizations, while recent studies, applying solid state HRM catheters, also reported the presence of simultaneous colonic pressure rises in children and adults (34, 41, 42). As regard to the patients with chronic constipation, the data of the present study are less suitable for comparison with those of Dinning et al (14) as these are conducted in different populations (we selected patients not responding to any available pharmacological treatments) and we also included bisacodyl stimulation.

The relative short duration of the recording period in the present study as compared to the 24hours measurements reported in literature $(21,26,28)$, as well as the fact that the measurements have been conducted after sedation and colonoscopy and in non-ambulatory subjects, are limitations which are common to most other colonic manometry studies $(13,14,25)$. Another potential limitation lies in the recording of the anal sphincter activity by means of $2.5 \mathrm{~cm}$ spaced sensors, which in theory may not allow the discrimination between real anal sphincter relaxations and movement of the catheter. However, the fact that the subjects did not move during the study, that the catheter was fixed to the buttock during the study, that the anal sphincter pressure decreases and spontaneously comes back to pre-relaxation pressure values all argue in favor of a physiologically relevant and accurate measure. Moreover a recent study only published in abstract form applying HRM to the anorectum has reported events with similar characteristics of rectal pressurizations and anal sphincter relaxations (43).

In conclusion, the present study demonstrated for the first time the occurrence of pan-colonic pressurizations associated with relaxations of the anal sphincter as a new colonic motor pattern which seems to be defective in patients with chronic constipation not responding to any pharmacological treatment. The mechanism behind the pan-colonic pressurizations as well as their function remains to be determined, but they are the main colonic motor pattern, increase during meal and with cholinergic stimulation. Pan-colonic pressurizations associated with relaxations of the anal sphincter may play a role in the transport of colonic gas and in the facilitation of the propagating sequences-induced colonic transport. 


\section{REFERENCES}

1. Longstreth GF, Thompson WG, Chey WD, et al. Functional bowel disorders. Gastroenterology 2006;130:1480-91.

2. Drossman DA, Li Z, Andruzzi E, et al. U.S. householder survey of functional gastrointestinal disorders. Prevalence, sociodemography, and health impact. Dig DisSci 1993;38:1569-80.

3. Wald A, Scarpignato C, Mueller-Lissner S, et al. A multinational survey of prevalence and patterns of laxative use among adults with self-defined constipation. Aliment Pharmacol Ther 2008;28:917-30.

4. Bampton PA, Dinning PG, Kennedy ML, et al. Spatial and temporal organization of pressure patterns throughout the unprepared colon during spontaneous defecation. Am $\mathrm{J}$ Gastroenterol 2000;95:1027-35

5. Cook IJ, Furukawa Y, Panagopoulos V, et al. Relationships between spatial patterns of colonic pressure and individual movements of content. Am J Physiol Gastrointest Liver Physiol 2000;278:G329-41.

6. Dinning PG, Zarate N, Hunt LM, et al. Pancolonic spatiotemporal mapping reveals regional deficiencies in, and disorganization of colonic propagating pressure waves in severe constipation. Neurogastroenterol Motil. 2010;22:e340-9.

7. Camilleri M, Bharucha AE, di Lorenzo C, et al. American Neurogastroenterology and Motility Society consensus statement on intraluminal measurement of gastrointestinal and colonic motility in clinical practice. Neurogastroenterol Motil. 2008; 20:1269-82.

8. Bharucha AE, Pemberton JH, Locke GR 3rd. American Gastroenterological Association technical review on constipation. Gastroenterology 2013;144:218-38.

9. Kahrilas PJ, Ghosh SK, Pandolfino JE. Esophageal motility disorders in terms of pressure topography: the Chicago Classification. J Clin Gastroenterol. 2008;42:627-35.

10. Kahrilas PJ, Bredenoord AJ, Fox M, et al. International High Resolution Manometry Working Group. The Chicago Classification of esophageal motility disorders, v3.0. Neurogastroenterol Motil. 2015;27:160-747.

11. Davidson JB, O'Grady G, Arkwright JW, et al. Anatomical registration and three-dimensional visualization of low and high-resolution pan-colonic manometry recordings. Neurogastroenterol Motil. 2011;23:387-90. 
12. Dinning PG, Wiklendt L, Gibbins I, et al. Low-resolution colonic manometry leads to a gross misinterpretation of the frequency and polarity of propagating sequences: Initial results from fiberoptic high-resolution manometry studies. Neurogastroenterol Motil. 2013;25:e640-9.

13- Dinning PG, Wiklendt L, Maslen L, et al. Quantification of in vivo colonic motor patterns in healthy humans before and after a meal revealed by high-resolution fiber-optic manometry. Neurogastroenterol Motil. 2014;26:1443-57.13.

14. Dinning PG, Wiklendt L, Maslen L, et al. Colonic motor abnormalities in slow transit constipation defined by high resolution, fibre-optic manometry. Neurogastroenterol Motil 2015;27:379-88.

15. Law NM, Bharucha AE, Undale AS, Zinsmeister AR. Cholinergic stimulation enhances colonic motor activity, transit, and sensation in humans. Am J Physiol Gastrointest Liver Physiol. 2001;281:G1228-37.

16. Corsetti M, Gevers AM, Caenepeel P, Tack J. The role of tension receptors in colonic mechanosensitivity in humans. Gut. 2004;53:1787-93.

17. M Corsetti, G Pagliaro, E Deloose, et al. Comparison of the effect of polyethylene glycol 3350 (PEG), prucalopride, bisacodyl and placebo on colonic motility assessed with intraluminal colonic high-resolution manometry in healthy subjects: the quantitative analysis. United European Gastroenterology Journal 2014; Issue 2, S1: A62.

18. Metcalf AM, Phillips SF, Zinsmeister AR, et al. Simplified assessment of segmental colonic transit. Gastroenterology. 1987;92:40-7.

19. Rao SS, Singh S, Sadeghi P. Is endoscopic mucosal clipping useful for preventing colonic manometry probe displacement? J Clin Gastroenterol. 2010; 44:620-4.

20. De Schryver AM, Samsom M, Smout AJ. In search of objective manometric criteria for colonic high-amplitude propagated pressure waves. Neurogastroenterol Motil. 2002; 14:375-81.

21. Rao SS, Sadeghi $P$, Beaty J, et al. Ambulatory 24-h colonic manometry in healthy humans. Am J Physiol Gastrointest Liver Physiol. 2001;280:G629-39.

22. Waldron DJ, Kumar D, Hallan RI, et al. Evidence for motor neuropathy and reduced filling of the rectum in chronic intractable constipation. Gut. 1990; 31:1284-8.

23. Enck P, Eggers E, Koletzko S, et al. Spontaneous variation of anal "resting" pressure in healthy humans. Am J Physiol. 1991;261:G823-6. 
24. O'Brien MD, Camilleri $M$, von der Ohe $M R$, et al. Motility and tone of the left colon in constipation: a role in clinical practice? Am J Gastroenterol. 1996;91:2532-8.

25. Daly J, Bergin A, Sun WM, et al. Effect of food and anti-cholinergic drugs on the pattern of rectosigmoid contractions. Gut. 1993;34:799-802.

26. Narducci F, Bassotti G, Gaburri M, et al. Twenty four hour manometric recording of colonic motor activity in healthy man. Gut. 1987;28:17-25.

27. Moreno-Osset E, Bazzocchi G, Lo S, et al. Association between postprandial changes in colonic intraluminal pressure and transit. Gastroenterology. 1989;96:1265-73.

28. De Schryver AM, Samsom M, Akkermans LM, et al. Fully automated analysis of colonic manometry recordings. Neurogastroenterol Motil. 2002;14:697-703.

29. Steadman CJ, Phillips SF, Camilleri M, et al. Variation of muscle tone in the human colon. Gastroenterology. 1991;101:373-81.

30. Rønholt C, Rasmussen OO, Christiansen J. Ambulatory manometric recording of anorectal activity. Dis Colon Rectum. 1999; 42:1551-9.

31. Ferrara A, Pemberton $\mathrm{JH}$, Levin KE, et al. Relationship between anal canal tone and rectal motor activity. Dis Colon Rectum. 1993; 36:337-42.

32. Rao SS, Sadeghi P, Beaty J, et al. Ambulatory 24-hour colonic manometry in slow-transit constipation. Am J Gastroenterol. 2004;99:2405-16.

33. Kamm MA, van der Sijp JR, Lennard-Jones JE. Observations on the characteristics of stimulated defaecation in severe idiopathic constipation. Int J Colorectal Dis. 1992;7:197-201.

34. Giorgio V, Borrelli O, Smith VV, et al. High-resolution colonic manometry accurately predicts colonic neuromuscular pathological phenotype in pediatric slow transit constipation. Neurogastroenterol Motil. 2013;25:70-8.

35. Spencer NJ, Hennig GW, Smith TK. A rhythmic motor pattern activated by circumferential stretch in guinea-pig distal colon. J Physiol. 2002;545:629-48.

36. Carbone SE, Dinning PG, Costa M, et al. Ascending excitatory neural pathways modulate slow phasic myogenic contractions in the isolated human colon. Neurogastroenterol Motil. 2013;25:6706. 
37. Spencer NJ, Kyloh M, Wattchow DA, et al. Characterization of motor patterns in isolated human colon: are there differences in patients with slow-transit constipation? Am J Physiol Gastrointest Liver Physiol. 2012;302:G34-43.

38. Mañé N, Martínez-Cutillas M, Gallego D, Jimenez M. Enteric motor pattern generators involve both myogenic and neurogenic mechanisms in the human colon. Front Physiol. 2015;6:205.

39. Bassotti G, Germani U, Morelli A. Flatus-related colorectal and anal motor events. Dig Dis Sci. 1996; 41:335-8.

40. Bendezú RA, Barba E, Burri E et al. Intestinal gas content and distribution in health and in patients with functional gut symptoms. Neurogastroenterol Motil. 2015;27:1249-57.

41. Wessel S, Koppen IJ, Wiklendt L, Costa M, Benninga MA, Dinning PG. Characterizing colonic motility in children with chronic intractable constipation: a look beyond high-amplitude propagating sequences. Neurogastroenterol Motil. 2016;28(5):743-57.

42. Chen $\mathrm{J}-\mathrm{H}, \mathrm{Yu} \mathrm{Y}, \mathrm{Yang} \mathrm{Z}$ et al. Colonic motor patterns in patients with chronic constipation assessed by high resolution manometry. Gastroenterology 2014; 146: S353.

43. Carrington EV, Hobson A, Knowles C, et al. Characterization of transient anal sphincter relaxations (TASRs) in health using high resolution anorectal manometry: redefining the sampling reflex? Neurogastroenterol Mot 2012; 24,s2:38.

\section{FIGURE LEGENDS}

Figure 1. Example of three pan-colonic pressurizations associated with anal sphincter relaxation in one subject at the color plot (left panel) and at the line plot (right panel). In the line plot panels the channels recording the activity of the anal sphincter are visualized in red.

Figure 2. Example of repetitive pan-colonic pressurizations associated with anal sphincter relaxation during a period of 20 minutes in fasting condition in one subject in the color plot (upper panel) and in the line plot (lower panel). In the line plot panels the channels recording the activity of the anal sphincter are visualized in red.

Figure 3. VAS score for the sensations reported by the subjects during the period they were awake (two hours before the meal and until the end of the recording) (A). Relationship between the number of pan-colonic pressurizations occurring every 15 min and the VAS score for the sensation of feeling of abdominal gas and desire to evacuate gas reported by the subjects during the period they awaked (B). 
Figure 4. A. Example of an episode of strain and of a pan-colonic pressurization in one subject. The channels recording the activity of the anal sphincter and of the EMG abdominal wall are visualized respectively in red and pink. B. Example of two episodes of movement artefacts, of two episodes of strain and of a pan-colonic pressurization in one subject. Note the concurrent increase in anal sphincter pressure and EMG abdominal wall activity during the episode of strain and of movement artifact and the presence of anal sphincter relaxation in absence of EMG activity during the pan-colonic pressurization.

Figure 5. A. Frequency of pan-colonic pressurizations during recovery, basal and after prostigmine periods. Data have been corrected for the different durations of recording periods. ${ }^{*} p=0.01$ vs basal period. B. Example of part of tracing before and after prostigmine in one subject. In color plot the markers in the upper part of the tracing identify the occurrence of sensations. In the line plot the channels recording the activity of the anal sphincter are visualized in red. Note the occurrence of repetitive pan-colonic pressurizations associated with anal sphincter relaxations and with sensations after the administration of prostigmine.

Figure 6. Response to intra-colonic administration of bisacodyl in color (upper panel) and in line (lower panel) plot in four patients. A. Note the occurrence of two HAPSs involving only the proximal part of the colon associated with apparent inhibition of the anal sphincter activity during the period before the occurrence of the propagating sequences. B Note the occurrence of repetitive pancolonic pressurizations associated with partial expulsion of the catheter. C. Note the occurrence of repetitive distal pan-colonic pressurizations. D. Note the occurrence of four HAPSs in the proximal part of the colon ending in the first case with a pan-colonic pressurization and in the other cases with repetitive pan-colonic pressurizations and partial expulsion of the catheter. 